\title{
Classification of Alzheimer's Disease from 3D Structural MRI Data
}

\author{
Nabil Belmokhtar \\ SIMPA Laboratory, Department of Informatics, \\ Faculty of Science, University of Sciences and \\ Technology of Oran - Mohamed Boudiaf. \\ P.B. 1505 El M'naouer, 31000 Oran, Algeria.
}

\author{
Nacéra Benamrane \\ SIMPA Laboratory, Department of Informatics, \\ Faculty of Science, University of Sciences and \\ Technology of Oran - Mohamed Boudiaf. \\ P.B. 1505 El M'naouer, 31000 Oran, Algeria.
}

\begin{abstract}
With nearly 100.000 cases in Algeria, Alzheimer's disease (AD) represents a major public health problem. Therefore, several different automated methods have been developed to assist clinicians in their diagnosis. We propose here a method based on binary support vector machines (SVM) to distinguish between patients with Alzheimer disease (AD), patients with mild cognitive impairment (MCI) and elderly control subjects (CS) from magnetic resonance imaging (MRI) data. In order to reduce the total computation time, we used the JADE (Java Agent DEvelopement Framework) multi-agent platform. The results obtained show the efficiency of our method and the significant advantages of the parallelization.
\end{abstract}

\section{General Terms}

Dementia Diagnosis, Neuroimaging, Supervised Learning, Multi-Agent Technology.

\section{Keywords}

Alzheimer's Disease Diagnosis, MRI, SVM Classification, Multi-Agent Systems, JADE.

\section{INTRODUCTION}

Alzheimer's disease (AD) is a neurodegenerative disease of the brain tissue that causes progressive and irreversible loss of mental functions. According to current epidemiological data, the $\mathrm{AD}$ affects about 26 million people worldwide, including 100.000 in Algeria [1]. Anatomical magnetic resonance imaging (MRI) studies have indicated that gray matter (GM) volume decreases with age while cerebrospinal fluid (CSF) increases. To provide appropriate care for Alzheimer's patients, it's very important to quantify the degree of atrophy in the cerebral cortex in the early stages of AD. In clinical practice, the medial temporal lobe atrophy (MTA) can be assessed visually. However, visual assessment is subjective and doesn't provide a true quantification of atrophy. For this purpose, various automated analysis methods have been developed [2] among which we can mention hippocampal volume measurement [3] and volume measurement of gray matter structures or cortical thickness measurement [4]. MTA is a sensitive marker for $\mathrm{AD}$, but not specific. It has been reported in other dementias including Parkinson's disease dementia (PDD), dementia with Lewy bodies (DLB) and vascular dementia (VD). Whole-brain methods [5] [6] for characterizing brain atrophy may be therefore more appropriate for differentiating $\mathrm{AD}$ from other neurodegenerative dementias. In particular, voxel-based morphometry (VBM) [7] [8] has become more widespread in the study of groups of healthy elderly, mild cognitive impairment (MCI) and $\mathrm{AD}$ subjects. To be useful, such methods must provide an individual predictive diagnosis. Many classification methods have been developed that allow an individual class prediction. Among the most popular: linear discriminant analysis (LDA) [9], neural network (NN) [10] and support vector machines (SVM) [5] [6] [11] [12]. In this paper, our purpose is to develop an automated method, by combining multiple binary SVM-based classifiers, able to discriminate between patients with $\mathrm{AD}$, patients with $\mathrm{MCI}$ and elderly control subjects (CS) by using a whole-brain VBM analysis applied to MRI images from the OASIS database. For that we use the feature vector extraction technique reported in [13]. In addition to these features, we also use two neuropsychological tests (Mini Mental State Exam (MMSE) and Clinical Dementia Rating (CDR)) to improve the prediction accuracy. In order to reduce the total processing time, we integrate the JADE (Java Agent DEvelopement Framework) multi-agent platform into our application. The architecture of the system is presented here.

\section{MATERIALS AND METHODS}

\subsection{Subjects}

Forty-five subjects (aged 65 to 96 years) were selected from the open access series of imaging studies (OASIS) database (available at http://www.oasis-brains.org) [14], including fifteen subjects with $\mathrm{AD}$, fifteen subjects with $\mathrm{MCI}$ and fifteen healthy aged control subjects (CS). A summary of subject demographics and dementia status is shown in table 1.

Table 1. Summary of subject demographics and dementia status

\begin{tabular}{|c|c|c|c|}
\hline & AD & MCI & CS \\
\hline $\begin{array}{c}\text { Number of } \\
\text { subjects }\end{array}$ & 15 & 15 & 15 \\
\hline Age & $\begin{array}{c}78,8 \\
(69-96)\end{array}$ & $\begin{array}{c}76,26 \\
(66-87)\end{array}$ & $\begin{array}{c}75,86 \\
(65-89)\end{array}$ \\
\hline Education & $\begin{array}{c}2,66 \\
(1-5)\end{array}$ & $\begin{array}{c}2,46 \\
(1-4)\end{array}$ & $\begin{array}{c}2,93 \\
(1-5)\end{array}$ \\
\hline $\begin{array}{c}\text { Socioeconomic } \\
\text { status }\end{array}$ & $\begin{array}{c}2,93 \\
(1-5)\end{array}$ & $\begin{array}{c}3,06 \\
(1-4)\end{array}$ & $\begin{array}{c}2,66 \\
(1-4)\end{array}$ \\
\hline CDR $(1 / 2)$ & $14 / 1$ & 0,5 & 0 \\
\hline MMSE & $\begin{array}{c}21,73 \\
(15-28)\end{array}$ & $\begin{array}{c}25,86 \\
(20-30)\end{array}$ & $\begin{array}{c}29,33 \\
(28-30)\end{array}$ \\
\hline
\end{tabular}

\subsection{Structural MRI Scanning Protocol}

For each subject, multiple (three or four) high-resolution structural 3D MRI scans using a T1-weighted magnetization- 
prepared rapid gradient echo (MP-RAGE) sequence were acquired on a 1.5-T Vision scanner (Siemens, Erlangen, Germany) in a single imaging session. MRI acquisition details: repetition time $\mathrm{TR}=9.7 \mathrm{msec}$, echo time $\mathrm{TE}=4.0$ msec., flip angle $\mathrm{FA}=10$, inversion time $\mathrm{TI}=20 \mathrm{msec}$., delay time TD= 200 msec., 128 sagittal $1.25 \mathrm{~mm}$ slices without gaps and pixels resolution of $256 \times 256(1 \times 1 \mathrm{~mm})$. An example of typical images is illustrated in figure 1 .
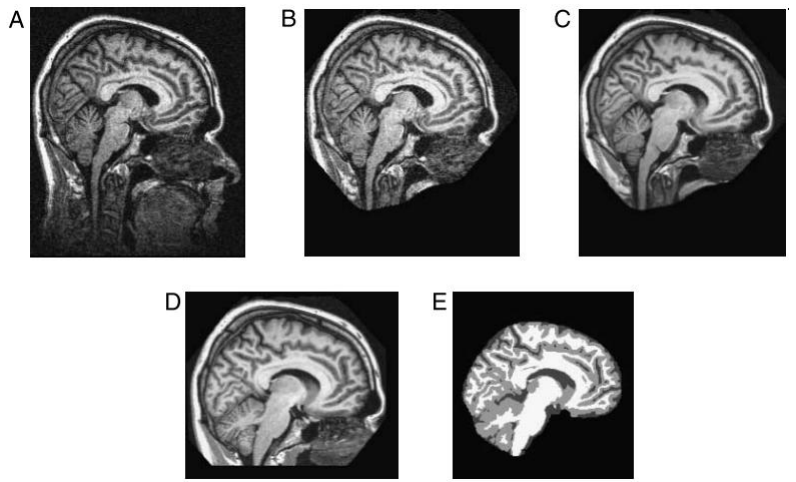

Fig 1: Typical MRI data set. (A) Individual scan before defacing. (B) Same scan after defacing. Note that the defacing process leaves the cranial vault intact while identifying facial features are removed. (C) Averaged motion-corrected image. Note improved signal-to-noise ratio. (D) Atlas-registered gain-field-corrected image. (E) Tissue classification image [14]

\subsection{Voxel-based Morphometry (VBM)}

Over the past several years, morphometry analysis has become an increasingly common tool for computational brain anatomy studies. It allows a comprehensive measurement of longitudinal (within the same brain, at different times) or cross-sectional (across brains) structural changes, not only in specific structures but throughout the whole-brain. Voxelbased morphometry (VBM) is a fully automated method that detects differences in the local composition of brain tissue on a voxel-wise comparison of multiple brain images [7] [8] [15]. Such technique has been applied to detect grey matter volume loss in Alzheimer's disease [6] [16] [17]. VBM analysis includes two steps: spatial preprocessing (normalization, segmentation, modulation and smoothing) and statistical analysis (voxel-wise statistical tests). In our case, both steps were implemented in the SPM8 software package [18] (running on Matlab 7.7) in order to obtain the classification features.

\subsection{Feature Extraction}

This section describes the feature vector extraction processes, based on the voxel location clusters detected by VBM analysis. The first process [13] computes the mean and standard deviation (MSD) of the GM voxel values of each voxel location cluster. The datasets are available at the following address:

http://www.ehu.es/ccwintco/uploads/8/85/Alzheimer_OASIS_ VBM _data.zip. These vectors were used as inputs to our classifier. In addition to these features, we also tried two other features: MMSE and CDR. The combination of these neuropsychological tests and structural MRI data can significantly improve the prediction performance.

\subsection{SVM Classification}

The classification is performed using SVM-type classifier. The SVM is a powerful supervised learning tool that combines high accuracy with good generalization capability. Several studies [5] [6] [11] [12] have demonstrated the great potential of using SVM to detect Alzheimer's disease. However, training SVM with multi-classes on a large data set is still a bottle-neck. Such harsh conditions increase the processing time necessary to train the algorithm and to make a decision. Therefore, SVM are convenient for classification problem with small-size data sets. To overcome these limitations, the distribution of calculations over several machines or processing units is necessary. The methodology is based on the idea of decomposing a problem into independent sub-problems running in parallel (simultaneously) and then coordinating their solution to solve the original problem. This can be achieved by using artificial intelligent techniques, such as multi-agent systems (MAS). One of the advantages of this approach is the availability of free software development platforms such as JADE (Java Agent DEvelopement Framework) allowing the developer to focus on the framework design instead of the programmatic details.

\subsection{JADE Multi-agent Platform}

JADE is a Java-based software framework that simplifies the implementation of multi-agent systems according to the FIPA-ACL standard [19]. It includes two basic parts: a FIPAcompliant agent platform and software package for the development of agents in Java. The choice of the Java programming language is justified by the fact that it allows a perfect object-oriented programming within distributed heterogeneous environments, plus some other features such as object serialization, reflection API and remote method invocation (RMI).

\section{SYSTEM ARCHITECTURE}

The system developed allows in a short time to categorize patients into one of these three groups: AD, MCI or CS. For that it uses a multi-class predictor combining three binary SVM-based classifiers. The algorithm is divided into several steps that can be performed by distinct agents allowing parallel computation. To ensure parallelization and coordination between agents, a multi-agent system was developed using the JADE platform.

\subsection{Prediction Algorithm}

The SVM is well known to be a binary classifier, which is usually trained on data from two classes. To deal with a multiclass datasets (three classes: $\mathrm{AD}, \mathrm{MCI}, \mathrm{CS}$ ), it is common to construct a set of binary classifiers, each trained by a portion of training dataset. Papers [20] [21] shows two popular methods: one-versus-all method using winner-takesall strategy (WTA SVM) and one-versus-one method implemented by max-wins voting (MWV SVM). For problems with $k$ classes, the first method trains $\mathrm{k}$ binary classifies each separating one class from the rest and then the multiclass classification is carried out according to the maximal output of the binary classifiers. On the other hand, in the one-versus-one method, $k(k-1) / 2$ binary classifiers are trained to distinguish one class from another. Given a new instance, the prediction is achieved by evaluating all $k(k-1) / 2$ individual classifiers and assigning the instance to the class with the highest number of votes. The prediction algorithm architecture developed here follows the one-versus-one strategy as described in figure 2 . 


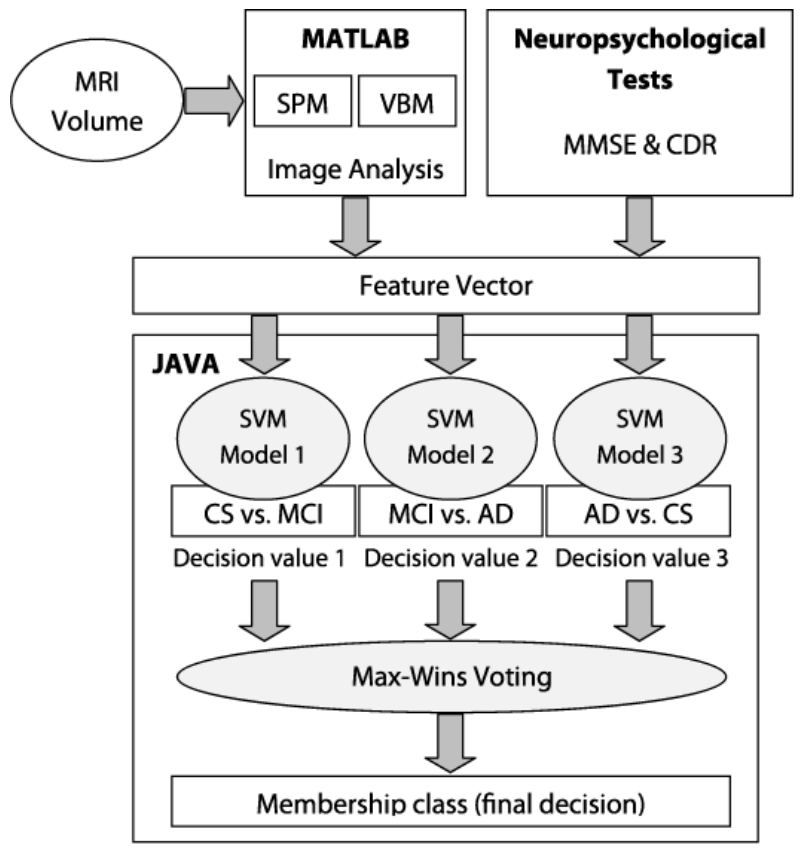

Fig 2: The prediction algorithm

\subsection{Multi-agent Implementation}

As mentioned before, we used the JADE multi-agent platform. JADE provides a distributed runtime environment, the agent and behavior (a task executed by an agent) abstractions, peer to peer communication and discovery mechanisms. As shown in figure 3 our system is composed of:

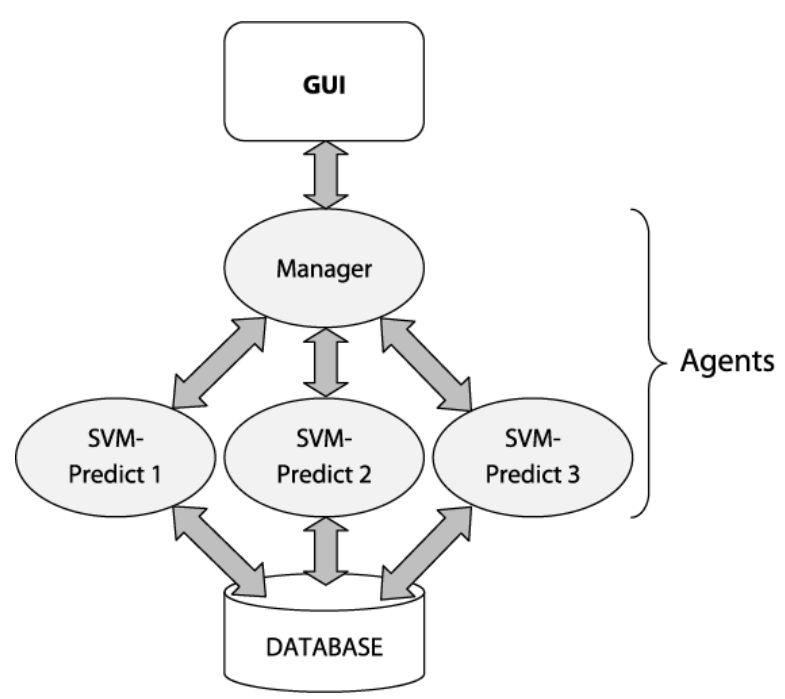

Fig 3: The multi-agent system architecture

\subsubsection{Graphical user interface (GUI)}

It allows the user to interact with the system, to check the input data and to visualize the results. The data is then transmitted to the Manager to start the prediction process of the introduced feature vector.

\subsubsection{Manager}

As its name suggests, this agent manages the prediction process. Ones the decision values obtained from each $S V M$ Predict agent, it returns the membership class (AD, MCI or CS) and display results in the $G U I$.

\subsubsection{SVM-Predict}

This agent calculates the decision value from the binary SVM-based classifier. Our system consists of three SVMPredict agents related to the total number of pairwise classifiers.

\section{RESULTS AND DISCUSSION}

We used the 5-fold cross-validation test to evaluate the performance of each binary SVM. For subjects, in each of the three sub-databases (CS vs. MCI, MCI vs. $A D$ and $A D$ vs. $\mathrm{CS}$ ), we first mix up all the training and test sets together. Then we randomly pick up 5 subjects as test data and the rest (25 subjects) as train data. We also estimated the classification accuracy rate defined as the ratio of the number of correctly classified instances to the total number of instances in the test set. On the other hand, we experimentally compared the performance between different SVM kernels and between feature extraction processes described in section 2.4. Tables 2, 3,4 and 5 show the classification results of the three binary SVMs (CS/MCI, MCI/AD and AD/CS).

For our feature extraction process, the best performance results were obtained with polynomial kernel of degree 3 , but are not far away from the results of the linear SVM. Experiments show also that the best accuracy of the algorithm can reach $1001 \%$ by using our feature extraction method.

Table 2. Classification results with a linear kernel

\begin{tabular}{|c|c|c|c|c|c|}
\hline \multicolumn{2}{|c|}{$\begin{array}{c}\text { SVM Agent } 1 \\
15 \text { CS vs. } 15 \text { MCI } \\
\end{array}$} & \multicolumn{2}{|c|}{$\begin{array}{c}\text { SVM Agent } 2 \\
15 \text { MCI vs. } 15 \text { AD } \\
\end{array}$} & \multicolumn{2}{|c|}{$\begin{array}{c}\text { SVM Agent } 3 \\
15 \text { AD vs. } 15 \text { CS }\end{array}$} \\
\hline 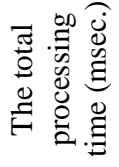 & 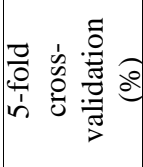 & 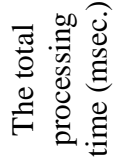 & 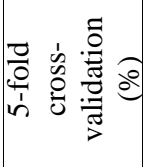 & 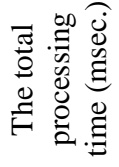 & 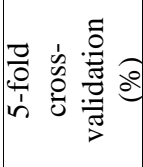 \\
\hline 187 & 93.33 & 187 & 100 & 188 & 100 \\
\hline
\end{tabular}

Table 3. Classification results with a polynomial kernel of degree 3

\begin{tabular}{|c|c|c|c|c|c|}
\hline \multicolumn{2}{|c|}{$\begin{array}{c}\text { SVM Agent } 1 \\
15 \text { CS vs. } 15 \text { MCI } \\
\end{array}$} & \multicolumn{2}{|c|}{$\begin{array}{c}\text { SVM Agent } 2 \\
15 \text { MCI vs. } 15 \text { AD } \\
\end{array}$} & \multicolumn{2}{|c|}{$\begin{array}{c}\text { SVM Agent } 3 \\
15 \text { AD vs. } 15 \text { CS }\end{array}$} \\
\hline 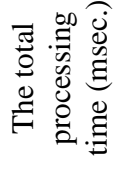 & 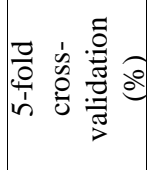 & 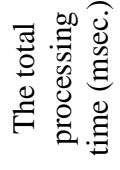 & 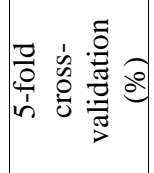 & 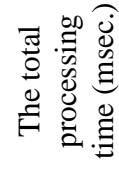 & 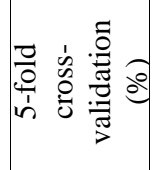 \\
\hline 187 & 100 & 218 & 100 & 187 & 100 \\
\hline
\end{tabular}

Finally, we evaluated the performance of our prediction algorithm using 98 subjects. Data from 45 subjects (15 CS, 15 MCI and $15 \mathrm{AD}$ ) were used for training. The remaining independent samples of 53 subjects (34 CS, $16 \mathrm{MCI}$ and 03 AD) were used to estimate the performance of our system. The sets of feature vectors used here correspond to our feature extraction process (MSD, MMSE and CDR). 
Table 4. Classification results with a RBF kernel

\begin{tabular}{|c|c|c|c|c|c|}
\hline \multicolumn{2}{|c|}{$\begin{array}{c}\text { SVM Agent } 1 \\
15 \text { CS vs. } 15 \text { MCI }\end{array}$} & \multicolumn{2}{|c|}{$\begin{array}{c}\text { SVM Agent } 2 \\
15 \text { MCI vs. } 15 \text { AD }\end{array}$} & \multicolumn{2}{|c|}{$\begin{array}{c}\text { SVM Agent } 3 \\
15 \text { AD vs. } 15 \text { CS }\end{array}$} \\
\hline 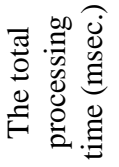 & 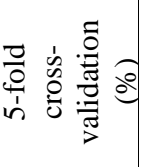 & 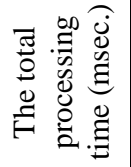 & 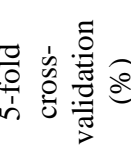 & 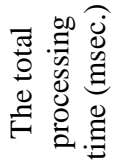 & 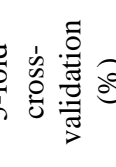 \\
\hline 188 & 93.33 & 188 & 73.33 & 187 & 96.66 \\
\hline
\end{tabular}

Table 5. Classification results with a sigmoid kernel

\begin{tabular}{|c|c|c|c|c|c|}
\hline \multicolumn{2}{|c|}{$\begin{array}{c}\text { SVM Agent } 1 \\
15 \text { CS vs. } 15 \text { MCI }\end{array}$} & \multicolumn{2}{|c|}{$\begin{array}{c}\text { SVM Agent } 2 \\
15 \text { MCI vs. } 15 \text { AD }\end{array}$} & \multicolumn{2}{|c|}{$\begin{array}{c}\text { SVM Agent } 3 \\
15 \text { AD vs. } 15 \text { CS }\end{array}$} \\
\hline 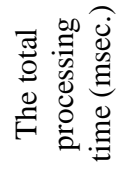 & 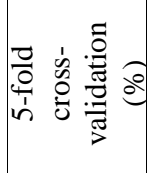 & 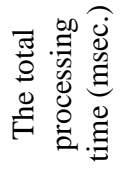 & 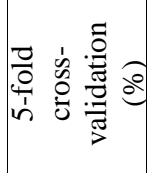 & 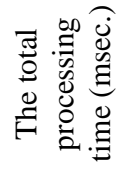 & 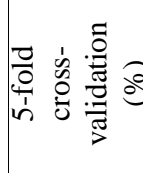 \\
\hline 187 & 50 & 187 & 50 & 187 & 50 \\
\hline
\end{tabular}

The table below shows that the best accuracy of the whole algorithm (100\%) was achieved when using our feature extraction process for SVM with polynomial kernel of degree 3 . On the other hand, the proposed method gives good speedup in term of total time required for training, testing and evaluation.

Table 6. Accuracy results of the prediction algorithm

\begin{tabular}{|c|c|c|}
\hline SVM kernel & $\begin{array}{c}\text { Global accuracy } \\
(\%)\end{array}$ & $\begin{array}{c}\text { The total processing } \\
\text { time (msec.) }\end{array}$ \\
\hline Linear & 84,9 & 178 \\
\hline Polynomial & 100 & 125 \\
\hline RBF & 62,26 & 109 \\
\hline Sigmoid & 7,54 & 109 \\
\hline
\end{tabular}

\section{CONCLUSION}

In this paper, we presented an automated method for classification of $\mathrm{AD}, \mathrm{MCI}$ and controls subjects from 3D structural MRI data sets. The classifier developed is based on SVM binary models using data analyzed by combining the VBM approach and neuropsychological tests to categorize the three groups of subjects. To reduce the total processing time, we used the JADE multi-agent platform. The results obtained were very satisfactory in terms of both accuracy and computational speed. It would be interesting to see how the results differ by using other feature selection methods and large MRI data sets. There are also some other possible extensions such as increasing the number of classes, increasing the number of agents involved in computational tasks and using another method for the final prediction step.

\section{REFERENCES}

[1] M. Zenati, (22/09/2011) "Maladie d'Alzheimer", Journal El-Moudjahid, $N^{\circ} 14313$ Nouvelle Série, ISSN 11110287, page 11, http://www.elmoudjahid.com/
[2] R. Cuingnet, E. Gerardin, J. Tessieras, G. Auzias, S. Lehéricy, M-O. Habert, M. Chupin, H. Benali, O. Colliot $\&$ The Alzheimer's Disease Neuroimaging Initiative, (2011) "Automatic classification of patients with Alzheimer's disease from structural MRI: A comparison of ten methods using the ADNI database", NeuroImage, 6 (2): 766-781.

[3] E. Ceyhan, C. Ceritoglu, M. F. Beg, L. Wang, J. C. Morris, J. G. Csernansky, M. I. Miller \& J. T. Ratnanather, (2008) "Analysis of metric distances and volumes of hippocampi indicates different morphometric changes over time in dementia of alzheimer type and nondemented subjects", Technical Report \# KU-EC-083, arXiv:0806.1473v2.

[4] O. Querbes, F. Aubry, J. Pariente, J. A. Lotterie, J. F. Demonet, V. Duret, M. Puel, I. Berry, J. C. Fort \& P. Celsis, (2009) "Early diagnosis of alzheimer's disease using cortical thickness: impact of cognitive reserve", Brain, 132(8):2036-2047.

[5] B. Magnin, L. Mesrob, S. Kinkingnehun, M. PelegriniIssac, M. Sarazin, B. Dubois, S. Lehericy \& H. Benali, (2009) "Support vector machine-based classification of Alzheimer's disease from whole-brain anatomical MRI", Neuroradiology, 51(2):73-83.

[6] L. Mesrob, B. Magnin, O. Colliot, M. Sarazinb, V. HahnBarma, Bruno, P. Gallinari, S. Lehéricy, S. Kinkingnéhun \& H. Benali. (2009) "Identification of Atrophy Patterns in Alzheimer's Disease based on SVM Feature Selection and Anatomical Parcellation", Annals of the BMVA Vol. 2009, No. 7, pp 1-9.

[7] J. Ashburner \& K. J. Friston, (2000) "Voxel-Based Morphometry - The Methods. NeuroImage", 11(6):805821 .

[8] A. Mechelli, C. J. Price, K. J. Friston \& J. Ashburner, (2005) "Voxel-Based Morphometry of the Human Brain: Methods and Applications", Current Medical Imaging Reviews, Vol. 1, No. 1, pp 105-113.

[9] B. M. French, M.R. W. Dawson \& Allen R. Dobbs, (1997) "Classification and staging of dementia of the Alzheimer type: a comparison between neural networks and linear discriminant analysis", Archives of. Neurology, 54(8):1001-1009.

[10] A. Savio, M. García-Sebastián, C. Hernández, M. Graña, \& J. Villanúa, (2009) "Classification Results of Artificial Neural Networks for Alzheimer's Disease Detection", Intelligent Data Engineering and Automated LearningIDEAL, Emilio Corchado, Hujun Yin (Eds) LNCS 5788, pages 641-648.

[11] P. P. M. Oliveira Jr., R. Nitrini, G. Busatto, C. Buchpiguel, J.R. Sato \& E. Amaro Jr., (2010) "Use of SVM Methods with Surface-Based Cortical and Volumetric Subcortical Measurements to Detect Alzheimer's Disease", Journal of Alzheimer's Disease 19:1263-1272.

[12] A.Savio, M. Graña \& J. Villanúa, (2011) "Deformation based features for Alzheimer's disease detection with linear SVM", HAIS (2): 336-343.

[13] D. Chyzhyk \& A. Savio. (2010) "Feature Extraction from Structural MRI Images Based on VBM: Data from OASIS Database", Technical Report GIC-UPV-EHU- 
RR-2010-10-14, Grupo de Inteligencia Computacional UPV/EHU.

[14] D. S. Marcus, T. H. Wang, J. Parker, J. G. Csernansky, J. C. Morris, \& R. L. Buckner, (2007) "Open Access Series of Imaging Studies (OASIS): Cross-sectional MRI Data in Young, Middle aged, Nondemented, and Demented Older Adults", Journal of Cognitive Neuroscience, 19(9):1498-1507, PMID: 17714011.

[15] J. L. Whitwell, (2009) "Voxel-Based Morphometry : An Automated Technique for Assessing Structural Changes in the Brain", The Journal of Neuroscience, 29(31):9661-9664.

[16] G. B. Frisoni, C. Testa, A. Zorzan, F. Sabattoli, A. Beltramello, H. Soininen, \& M. P. Laakso, (2002) "Detection of Grey Matter Loss in Mild Alzheimer's Disease with Voxel Based Morphometry", Journal of Neurology, Neurosurgery \& Psychiatry, 73(6):657-664.

[17] G. F. Busatto, G. E. J. Garrido, O. P. Almeida, C. C. Castro, C. H. P. Camargo, C. G. Cid, C. A. Buchpiguel,
S. Furuie \& C. M. Bottino, (2003) “A Voxel-Based Morphometry Study of Temporal Lobe Gray Matter Reductions in Alzheimer's Disease". Neurobiology of Aging, 24(2):221-231.

[18] J. Ashburner, G. Barnes, C-C. Chen... (2011) "SPM8 Manual (Statistical Parametric Mapping)", Functional Imaging Laboratory, Wellcome Trust Centre for Neuroimaging, London, UK, http://www.fil.ion.ucl.ac.uk/spm/

[19] F. Bellifernine, G. Rimassa \& A. Poggi, (1999) "JADE A FIPA-compliant Agent Framwork", PAAM99, http://jade.tilab.com/

[20] K-B. Duan \& S. S. Keerthi, (2005) "Which Is the Best Multiclass SVM Method? An Empirical Study”, Lecture Notes in Computer Science, vol. 3541, pp. 278-285.

[21] C.-W. Hsu \& C.-J. Lin, (2002) “A comparison of methods for multi-class support vector machines", IEEE Transactions on Neural Networks, Vol. 13, pp. 415-425. 